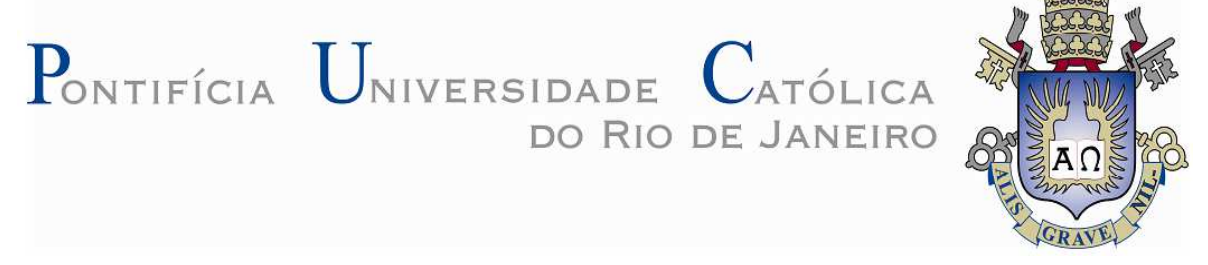

José Eduardo Moreno Léverenz

\title{
Simulação de Fluxo em Reservatórios Não Convencionais de Folhelhos
}

Dissertação apresentada como requisito parcial para obtenção do grau de Mestre pelo Programa de Pós-Graduação em Engenharia Civil do Departamento de Engenharia Civil da PUC-Rio.

Orientador: Prof. Sérgio Augusto Barreto da Fontoura Co-orientador: Dr. Nelson Inoue 


\section{Pontifícia Universidade Católica $_{\text {a }}$ \\ DO RIO DE JANEIRO

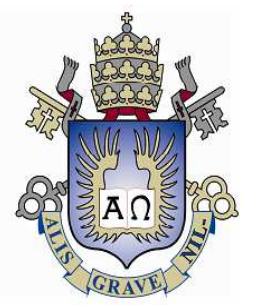

José Eduardo Moreno Léverenz

\section{Simulação de Fluxo em Reservatórios Não Convencionais de Folhelhos}

Dissertação apresentada como requisito parcial para obtenção do grau de Mestre pelo Programa de Pós-Graduação em Engenharia Civil do Departamento de Engenharia Civil do Centro Técnico Científico da PUC-Rio. Aprovada pela Comissão Examinadora abaixo assinada.

Prof. Sérgio Augusto Barreto da Fontoura Orientador

Departamento de Engenharia Civil - PUC-Rio

Prof. Celso Romanel

Departamento de Engenharia Civil - PUC-Rio

Prof. Eurípides do Amaral Vargas Junior Departamento de Engenharia Civil - PUC-Rio

Prof. Luis Glauber Rodrigues

Departamento de Engenharia Mecânica - PUC-RIO

Prof. José Eugenio Leal Coordenador Setorial do Centro Técnico Científico - PUC-RIO

Rio de Janeiro, 15 de Abril de 2015 
Todos os direitos reservados. É proibida a reprodução total ou parcial do trabalho sem autorização da universidade, do autor e dos orientadores.

\section{José Eduardo Moreno Léverenz}

Graduou-se em Engenharia Civil pela Universidade Nacional de San Antonio Abad del Cusco (Cusco-Peru) em 2008. Trabalhou com projetos rodoviários no Peru no período 2008-2012. Ingressou no mestrado de engenharia civil em 2013, na Pontifícia Universidade Católica do Rio de Janeiro.

Ficha Catalográfica

Moreno Léverenz, José Eduardo

Simulação de fluxo em reservatórios não convencionais de folhelhos / José Eduardo Moreno Léverenz; orientador: Sérgio Augusto Barreto da Fontoura; co-orientador: Nelson Inoue. - 2015.

(146) f. : il. (color.) ; $30 \mathrm{~cm}$

Dissertação (Mestrado) - Pontifícia Universidade Católica do Rio de Janeiro, Departamento de Engenharia Civil, 2015.

\section{Inclui bibliografia}

1. Engenharia civil - Teses. 2. Reservatórios não convencionais. 3. Folhelho. 4. Fraturamento hidráulico. 5. Dupla permeabilidade. I. Fontoura, Sérgio Augusto Barreto da. II. Inoue, Nelson. III. Pontifícia Universidade Católica do Rio de Janeiro. Departamento de Engenharia Civil. IV. Título. 


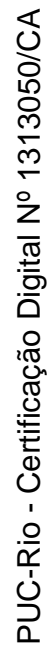

Dedico este trabalho aos meus pais:

Ester Léverenz Melena e Jorge Julio Moreno Reátegui 


\section{Agradecimentos}

Primeiramente agradeço a Deus, pelo amor, pela vida, pela saúde, por todas as coisas maravilhosas que já fez na minha vida. O senhor é quem guia meus passos e me sinto abençoado pelo seu amor infinito. Obrigado Senhor por todas as experiências vividas nestes dois anos de mestrado, por todas as pessoas maravilhosas que colocaste em meu caminho.

Agradeço a meus pais por todo o amor incondicional que sempre me deram, pelo apoio em cada momento da minha vida. Uma vida toda não é suficiente para expressar toda a gratidão que eu tenho por vocês. Tudo que sou devo a vocês.

Agradeço ao meu filho Franco, que é a maior motivação que eu tenho na minha vida para fazer as coisas corretamente. Agradeço a Milagros pela paciência e apoio que me brindou nestes dois anos.

Agradeço ao meu orientador, Prof. Sérgio Fontoura, por essa oportunidade de crescimento profissional e pessoal e pela sua excelente orientação deste trabalho. Obrigado Prof. Sérgio, pela confiança depositada no meu trabalho. Foi um grande aprendizado trabalhar com o senhor.

Agradeço ao meu co-orientador, Dr. Nelson Inoue, pelo apoio e incentivo, pelas sugestões fornecidas nos momentos oportunos. Fico muito grato por sua ajuda brindada.

Agradeço aos amigos da equipe do GTEP, Grupo de Tecnologia e Engenharia de Petróleo, localizada no Colégio São Marcelo: Guilherme, Carlos Emmanuel, Carla, Juan David, Rafael, Daniel, Ismael, Ronald, Laura, Dilia e Danilo, por me receberem muito bem, pelo companheirismo e incentivo no desenvolvimento deste trabalho.

Agradeço aos professores da pós-graduação da PUC-Rio, que em cada aula entregam mais do que conhecimento, entregam sua experiência e qualidade de pessoas, obrigado por tudo o ensinado nestes anos.

Agradeço a minha irmã Patricia, pelo seu apoio durante estes dois anos. Muito obrigado ao meu grande amigo, a quem considero como um irmão: Jordey, sou muito grato por toda ajuda que você me deu. Agradeço também aos meus sobrinhos: Jorge, Carlos e Claudia. A todos eles muito obrigado pela convivência familiar que me deram nestes dois anos.

Agradeço a meu irmão Julio, que é um grande exemplo para mim. Pela força que sempre me brindou, pelo incentivo e apoio para conseguir alcançar os objetivos propostos. 
Aos meus amigos: Juancito, Laura, Jeferson, Raúl, Eliot, Gustavo, Fernando, Adriano, Daniel, Natalia, Ronald, Rhaissa, Sandra, Jimmy e Dilia. Obrigado pela amizade. Eu quero agradecer de maneira muito especial ao meu amigo Francisco, pela grande ajuda que você me deu durante o mestrado.

Agradeço aos meus grandes amigos Robert e Geber obrigado pelas ligações que sempre fizeram para me dar uma força e motivar nos momentos que eu precisava.

Não posso deixar de agradecer a todos os funcionários do Departamento de Engenharia Civil da PUC-Rio, em especial a Rita Leite.

Agradeço à CAPES e à PUC-Rio, pelos auxílios concedidos, sem os quais este trabalho não poderia ter sido realizado. 


\section{Resumo}

Léverenz, José Eduardo Moreno; Fontoura, Sérgio Augusto Barreto da; Inoue, Nelson. Simulação de Fluxo em Reservatórios Não Convencionais de Folhelhos. Rio de Janeiro, 2015. 146 p. Dissertação de Mestrado. Departamento de Engenharia Civil, Pontifícia Universidade Católica do Rio de Janeiro.

Os reservatórios não convencionais de folhelho possuem permeabilidades muito baixas sendo necessário fraturar hidraulicamente a formação para aumentar a permeabilidade e conseguir um melhor desempenho do poço. $\mathrm{O}$ fraturamento hidráulico em vários estágios criado a partir de um poço horizontal gera uma complexa rede de fraturas, com variadas condutividades dentro do volume de reservatório estimulado. Neste trabalho realizamos análises paramétricas para avaliar os efeitos no desempenho do poço produtor causadas pela variação dos diferentes parâmetros dentro da complexa rede de fraturas criada. Estes parâmetros foram principalmente: os espaçamentos na rede de fraturas (sem propante), a condutividade na rede de fraturas (sem propante), a condutividade das fraturas primárias (preenchidas com propante), os espaçamentos das fraturas primárias (preenchidas com propante), a variação da permeabilidade ao longo das fraturas primárias e finalmente a variação das pressões do fundo do poço. Entender as relações entre o tamanho da rede de fraturas, os espaçamentos, a distribuição do propante e a condutividade das fraturas são parâmetros chaves para definir estratégias de estimulação e completação. Finalmente se fez uma simulação com injeção de água avaliando o impacto que causa na recuperação total de óleo e gás, e analisamos a possibilidade de aplicar este método de recuperação num reservatório fraturado hidraulicamente.

\section{Palavras-chave}

Reservatórios não convencionais; folhelho; fraturamento hidráulico; dupla permeabilidade. 


\section{Abstract}

Léverenz, José Eduardo Moreno; Fontoura, Sérgio Augusto Barreto da (Advisor); Inoue, Nelson (Co-Advisor). Flow Simulation in Unconventional Shale Reservoirs. Rio de Janeiro, 2015. 146 p. MSc. Dissertation - Departamento de Engenharia Civil, Pontifícia Universidade Católica do Rio de Janeiro.

The shale reservoirs have ultralow permeability requiring hydraulically fracturing the formation to improve permeability and get a better well performance. The multistage hydraulic fractures system created from a horizontal well generates a complex fracture network with different conductivities within the stimulated reservoir volume. In this work we made parametric analyzes to assess the effects on producer well performance caused by the variation of different parameters within the complex network fractures created. These parameters were mainly: spacing in the un-propped fracture network, the conductivity in the unpropped fracture network, primary fracture conductivity, primary fracture spacing, the variation of permeability along the primary fractures and finally the variation of downhole pressures. Understanding the relationship between fracture network size, fracture spacing, proppant distribution, and fracture conductivity is critical to stimulation and completion design. Finally a simulation with water injection evaluating its impact on recovering oil and gas was carried out, and the possibility of applying this method of recovery in a fractured reservoir was evaluated.

\section{Keywords}

Unconventional reservoirs; shale; fracking; dual permeability. 


\section{Sumário}

1. Introdução 18

1.1. Relevância e motivação do estudo 18

1.2. Objetivos 19

$\begin{array}{ll}\text { 1.3. Organização da dissertação } & 19\end{array}$

2. Revisão bibliográfica 21

2.1. Reservatórios de gás não convencionais 21

2.2. Shale gás 24

2.3. Oil shale e shale oil 25

2.4. Fraturamento hidráulico 26

2.5. Fraturamento hidráulico em vários estágios 29

2.6. Mapeamento micro sísmico (MS mapping) 31

2.7. Volume de reservatório estimulado (SRV) 34

2.8. Métodos de simulação de reservatórios naturalmente fraturados 38

2.8.1. Modelo de dupla porosidade 39

2.8.2. Modelo de dupla porosidade e dupla permeabilidade 42

2.8.3. Termo de transferência de fluxo entre a matriz e as fraturas $\quad 43$

3 . Simulação de fluxo em reservatórios de folhelho 44

3.1. Modelagem de fluxo em reservatórios fraturados 44

3.1.1. Dupla porosidade standard (DUALPOR) 45

3.1.2. Dupla permeabilidade (DUALPERM) 45

3.1.3. Subdomínio (SUBDOMAIN) 46

3.1.4. Multiple interacting continua (MINC) 47

3.2. Equações de conservação de massa 49

3.2.1. Transmissibilidade $\quad 50$

3.2.2. Coeficiente de transferência 51

3.3. Pseudo permeabilidade da fratura primária 51

3.4. Permeabilidade e porosidade da rede de fraturas 52

3.4.1. Permeabilidade da rede de fraturas 52 
3.4.2. Porosidade da rede de fraturas 53

3.5. Fluxo não darcy em reservatórios 54

3.6. Fator de correção do coeficiente não Darcy 55

4 . Simulação do modelo de reservatório 58

4.1. Descrição do simulador 58

4.2. Modelo base 58

4.2.1. Descrição do reservatório 59

4.2.2. Características da matriz e das fraturas 61

4.2.3. Propriedades dos componentes do fluido (PVT) 62

4.2.4. Propriedades rocha-fluido 64

4.2.5. Condições iniciais 68

4.3. Análise paramétrica 68

4.3.1. Caso 1: variação dos espaçamentos na rede de fraturas $\quad 69$

4.3.2. Caso 2: variação das condutividades na rede de fraturas $\quad 70$

4.3.3. Caso 3: variação das condutividades nas fraturas primárias $\quad 70$

4.3.4. Caso 4: variação dos espaçamentos nas fraturas primárias 71

4.3.5. Caso 5: Variação da permeabilidade ao longo da fratura primária 72

4.3.6. Caso 6: variação da pressão do fundo do poço 74

4.4. Modelo de injeção de água 74

5. Resultados e discussão 76

5.1. Resultados da análise paramétrica 76

5.2. Resultados do modelo de injeção de água 90

6. Considerações finais 95

6.1. Conclusões 95

6.2. Sugestões para trabalhos futuros 97

$\begin{array}{ll}\text { Referências bibliográficas } & 98\end{array}$

$\begin{array}{ll}\text { Anexo } & 102\end{array}$ 


\section{Lista de figuras}

Figura 2.1 Esquema geológico dos recursos de gás (Elgmati, 2011) 21

Figura 2.2 Recursos de hidrocarbonetos em todo o mundo

(Chen, 2013)

Figura 2.3 Pirâmide de recursos com foco em recursos não convencionais (Rajnauth, 2012)

Figura 2.4 Possíveis cenários da distribuição do propante (Cipolla, 2009)

Figura 2.5 Exemplos de possíveis tipos de fraturas

(Warpinski et al., 2008)

Figura 2.6 Fraturamento hidráulico multi fase (Wan, 2013)

Figura 2.7 Mapeamento micro sísmico poço vertical, Barnett Shale (Warpinski et al., 2008)

Figura 2.8 Eventos padrões MS para a Barnett Shale, fraturamento hidráulico num poço horizontal (Cipolla et al., 2009)

Figura 2.9 Relação da longitude total da rede de fraturas como função do volume total de fluido bombeado (Fisher et al., 2002)

Figura 2.10 Estimativa da SRA a partir dos dados de mapeamento micro sísmico (Mayerhofer et al., 2008)

Figura 2.11 Esboço conceitual do cálculo da altura SRV

(Mayerhofer et al., 2008)

Figura 2.12 SRV vs 15 anos de produção acumulada

(Mayerhofer et al., 2008)

Figura 2.13 Idealização das heterogeneidades do meio poroso

(Warren e Root, 1963)

Figura 2.14 Representação do reservatório fraturado no modelo de dupla porosidade. Destaque para a sobreposição dos grids de fratura e de matriz (Lemonnier e Bourbiaux, 2010)

Figura 2.15 Detalhe do bloco de matriz cercado por fraturas (Paiva, 2012)

Figura 3.1 Refinamento vertical do bloco da matriz 
(User's guide imex)

Figura 3.2 Refinamento aninhado do bloco da matriz

(User's guide imex)

Figura 3.3 Diagramas esquemáticos das conectividades

(User's guide imex)

48

Figura 4.1 Modelo base do reservatório com duas fraturas planares transversais ao poço horizontal 60

Figura 4.2 Poço produtor com duas fraturas primárias transversais $\quad 61$

Figura 4.3 Curvas de permeabilidade relativa da água e do óleo na matriz

65

Figura 4.4 Curvas de permeabilidade relativa do óleo e gás na matriz 66

Figura 4.5 Variação da pressão capilar óleo-água vs a saturação da água na matriz

Figura 4.6 Variação da pressão capilar óleo-gás vs a saturação do líquido na matriz

Figura 4.7 Curvas de permeabilidade relativa nas fraturas

Figura 4.8 Variação da permeabilidade nas fraturas primárias

Figura 4.9 Permeabilidades nas fraturas primárias e na matriz.

73

Figura 4.10 Modelo base de injeção de água 75

Figura 5.1 Efeito dos espaçamentos na rede de fraturas 77

Figura 5.2 Variações das pressões médias do reservatório 77

Figura 5.3 Efeitos das condutividades na rede de fraturas 79

Figura 5.4 Variações das pressões médias do reservatório 79

Figura 5.5 Efeitos das condutividades nas fraturas primárias 81

Figura 5.6 Variações das pressões médias do reservatório 81

Figura 5.7 Efeitos dos espaçamentos das fraturas primárias 83

Figura 5.8 Variações das pressões médias do reservatório 83

Figura 5.9 Pressões no reservatório ao longo do período de produção 85

Figura 5.10 Comparação das curvas de fator de recuperação de gás e pressão média do reservatório, para permeabilidades constantes e variadas ao longo das fraturas primárias 86

Figura 5.11 Fator de recuperação de óleo para diferentes BHP 87

Figura 5.12 Fator de recuperação de gás para diferentes BHP 
Figura 5.13 Variações das pressões para diferentes BHP

Figura 5.14 Curvas de pressão média do reservatório e injeção de água

Figura 5.15 Fator de recuperação de óleo para os diferentes cenários 91

Figura 5.16 Modelos de injeção de água no modelo base

Figura 5.17 Curvas de fator de recuperação de óleo, água acumulada e pressão do reservatório para o modelo com poço injetor na matriz

Figura 5.18 Curvas de fator de recuperação de óleo, água acumulada e pressão do reservatório para o modelo com poço injetor na fratura primária 


\section{Lista de tabelas}

Tabela 2.1 Resumo dos hidrocarbonetos convencionais e não convencionais por regiões no mundo

Tabela 3.1 Correlações para o fluxo não Darcy segundo Geertsma e Frederick \& Graves.

56

Tabela 4.1Caracteristicas de matriz e fraturas. 62

Tabela 4.2 Propriedades PVT do fluido. 63

Tabela 4.3 Propriedades adicionais do fluido. 64

Tabela 4.4 Casos avaliados na análise paramétrica. 69

Tabela 4.5 Variação dos espaçamentos na rede de fraturas. 69

Tabela 4.6 Variação das condutividades na rede de fraturas. 70

Tabela 4.7 Variação das condutividades nas fraturas primárias. 71

Tabela 4.8 Variação do espaçamento nas fraturas primárias

72

Tabela 5.1 Fator de recuperação de óleo para diferentes espaçamentos na rede de fraturas.

Tabela 5.2 Fator de recuperação de óleo para diferentes condutividades na rede de fraturas.

Tabela 5.3 Fator de recuperação de óleo para diferentes condutividades nas fraturas primárias.

Tabela 5.4 Fator de recuperação de óleo para diferentes espaçamentos de fraturas primárias.

Tabela 5.5 Fator de recuperação de óleo. 88

Tabela 5.6 Fator de recuperação de gás. 89 


\section{Lista de nomenclatura}

Letras Latinas:

A Área

B Fator volume de formação do fluido (ex. $\left.B_{0}, B_{w}, B_{g}\right)$

D ou Z Profundidade de análise

$\mathrm{E}_{\mathrm{g}} \quad$ Fator de expansão do gás (inverso do $\mathrm{B}_{\mathrm{g}}$ )

$\mathrm{ft} \quad$ Pés

$L_{x}, L_{y}, L_{z}$ Dimensões equivalentes do bloco de matriz

K Permeabilidade absoluta

k Permeabilidade efetiva

$\mathrm{k}_{\mathrm{r}} \quad$ Permeabilidade relativa

$\mathrm{K}_{\mathrm{fx}} \quad$ Permeabilidade da fratura na direção $\mathrm{x}$

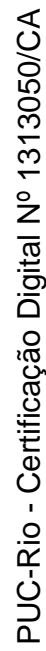

$\mathrm{K}_{\mathrm{fy}} \quad$ Permeabilidade da fratura na direção y

$\mathrm{K}_{\mathrm{fz}} \quad$ Permeabilidade da fratura na direção $\mathrm{z}$

$\mathrm{K}_{\text {feff }} \quad$ Permeabilidade da fratura nos blocos do modelo de $2 \mathrm{ft}$

$\mathrm{K}_{\text {corr }} \quad$ Permeabilidade da fratura corrigida nos blocos do modelo $2 \mathrm{ft}$

md Mili Darcy

nd Nano Darcy

$\mathrm{N} 1_{\alpha} \quad$ Parâmetro para determinar o coeficiente de fluxo não darcy

$\mathrm{N} 2_{\alpha} \quad$ Parâmetro para determinar o coeficiente de fluxo não darcy

p Pressão

$\mathrm{P}_{\mathrm{C}} \quad$ Pressão capilar

$\mathrm{R}_{\mathrm{S}} \quad$ Razão de solubilidade do gás no óleo

q Vazão de fluidos

S Saturação do fluido

t Tempo

$\mathrm{T} \quad$ Transmissibilidade

V Volume

Vis。 Viscosidade do óleo

Vis $_{\mathrm{g}} \quad$ Viscosidade do gás 
Letras gregas:

$\alpha \quad$ Parâmetro para determinar o coeficiente de fluxo não Darcy

$\gamma \quad$ Peso específico do fluido

$\beta \quad$ Coeficiente de fluxo não Darcy

$\Delta \mathrm{p} \quad$ Variação da pressão

$\Delta \mathrm{x}, \Delta \mathrm{y}, \Delta \mathrm{z}$ Dimensões de uma célula de simulação

$\nabla \quad$ Divergente

$\mu \quad$ Viscosidade dinâmica do fluido

$\rho \quad$ Densidade

$\sigma \quad$ Fator de forma (referente às dimensões do bloco de matriz)

$\tau \quad$ Função de transferência

$\Phi \quad$ Porosidade

$\Phi \quad$ Potencial de fluxo

$\checkmark \quad$ Velocidade

ঠ $\quad W_{f}$

Largo da fratura

Sobrescrito:

$\wedge \quad$ Representa os termos da fratura (ex. porosidade da fratura, $\hat{\phi}$ )

$x \quad$ Direção no sentido " $x$ "

n Nível de tempo antigo

$\mathrm{n}+1 \quad$ Nível de tempo novo

Subscrito:

$\begin{array}{ll}\alpha & \text { Fase (óleo, água ou gás) } \\ \mathrm{f} & \text { Fratura } \\ \mathrm{m} & \text { Matriz } \\ 0 & \text { Óleo } \\ \mathrm{g} & \text { Gás } \\ \mathrm{w} & \text { Água } \\ \mathrm{t} & \text { Igual a f ou m (fratura ou matriz) } \\ \mathrm{corr} & \text { Corrigido } \\ \mathrm{feff} & \text { Fratura efetiva } \\ \mathrm{i}+1 / 2 & \text { Fronteira entre duas células }\end{array}$


Siglas:

\begin{tabular}{|c|c|}
\hline AAO & Austral-Asia \\
\hline AFR & Africa \\
\hline bbl & Barril \\
\hline $\mathrm{BHP}$ & Pressão do fundo do poço \\
\hline CIS & Comunidade dos estados independentes \\
\hline CMG & Computer modelling group \\
\hline DIFRAC & Espaçamento de fraturas na direção "i" \\
\hline DJFRAC & Espaçamento de fraturas na direção “j” \\
\hline DUALPERM & Dupla permeabilidade \\
\hline DUALPOR & Dupla porosidade \\
\hline EUP & Europa \\
\hline IMEX & Implícito-Explícito \\
\hline LAM & Latino América \\
\hline MET & Médio oriente \\
\hline MINC & Multiple Interacting Continua \\
\hline NAM & América do norte \\
\hline SRV & Volume de reservatório estimulado \\
\hline SUBDOMAIN & Subdomínio \\
\hline tfc & Trilhões de pés cúbicos \\
\hline
\end{tabular}

